\title{
SOI DEVICE ISLANDS FORMED BY OXYGEN IMPLANTATION THROUGH PATTERNED MASKING LAYERS
}

\author{
U. Bussmann, A.K. Robinson and P.L.F. Hemment \\ Department of Electronic and Electrical Engineering \\ University of Surrey, Guildford GU2 5XH, UK \\ G.J. Campisi \\ Naval Research Labs, Washington, DC 20375, USA
}

\section{Introduction}

SIMOX (Separation by Implanted Oxygen) is recognized as a versatile SOI technology. Substrates are produced by implanting high doses of oxygen into silicon, followed by a high temperature annealing stage, in which the implanted oxygen segregates to form a buried stoichiometric $\mathrm{SiO}_{2}$ layer. Implantation dose and energy determine the layer thickness in the resulting structure. In conventional SIMOX technology the lateral isolation of device islands is achieved by local oxidation or mesa etching of the top silicon layer. This leads to a highly non-planar surface.

An alternative route to device islands utilizes masking layers during implantation [1]. Windows in the mask define the silicon island positions. In these regions the layer structure corresponds to the conventional SIMOX structure. However, in the mask region, where the oxygen ions loose part of their kinetic energy before reaching the silicon, the buried oxide is shifted towards the surface. The aim of this approach is to achieve total dielectric isolation by implantation and annealing only, thus avoiding a subsequent LOCOS or mesa etching step. New experimental parameters, which include masking material, mask thickness and the geometry of the bevel edge, determine the structurai properties of the non-planar oxide as well as the surface topology.

\section{Experimental}

Polycrystalline silicon, $\mathrm{SiO}_{2}$, and $\mathrm{Si}_{2} \mathrm{~N}_{4}$ have been used as masking materials on (100) silicon wafers. $200 \mathrm{keV} \mathrm{O}{ }_{2}^{+}$implantations were performed at $550^{\circ} \mathrm{C}$. The samples were annealed at $1310^{\circ} \mathrm{C}$ for 5 hours.

\section{Results and Discussion}

Polycrystalline silicon masks have been successfully used to form continuous non-planar buried oxide layers. As an example fig. 1 shows the result of a $1.8 \times 10^{13}$ $\mathrm{O}^{+} \mathrm{cm}^{-2}$ implantation through a $250 \mathrm{~nm}$ mask. As expected, after annealing dislocations are found in the silicon top layer and silicon inclusions in the oxide close to the lower interface. In addition a vertical chain of inclusions appears in the transition region. These inclusions might be avoided by decreasing the slope of the mask edge. A thin superficial silicon overlayer is left undemeath the mask, which can be used as an etch stop when removing the remainder of the masking material.

A thermal oxide mask is rapidly eroded during implantation (sputter coefficient 1.1 atoms/ion for molecular oxygen). Our computer simulation code IRIS [2] has been used to study the evolution of the oxygen implantation profile. In the masked region the peak of a small incremental dose, distribution is always located below the maximum position of the already implanted oxygen. The buried oxide layer is predominantly growing at the lower interface and the slope of the corresponding flank in the profile increases. 
Therefore, the buried layer formation mechanism differs from the conventional SIMOX situation. The formation of islands at the lower oxide interface can be avoided.

When the oxide mask is formed in a LOCOS process $55 \%$ of this masking layer is located above and $45 \%$ below the silicon surface level. As shown by simulations, a planar surface can then be achieved after implantation (see schematic in fig. 2). Also, the transition between the buried oxide layers in the mask and window regions will be smoother. Therefore, a structural improvement of the critical transition region is expected. TEM results of this new structure will be presented and discussed.

\section{References:}

[1] J.R. Davis, A.K. Robinson, K.J. Reeson and P.L.F. Hemment, Appl. Phys. Lett. 51 (1987) 1419

[2] U. Bussmann and P.L.F. Hemment, Nucl. Instr. and Meth. B47 (1990) 22

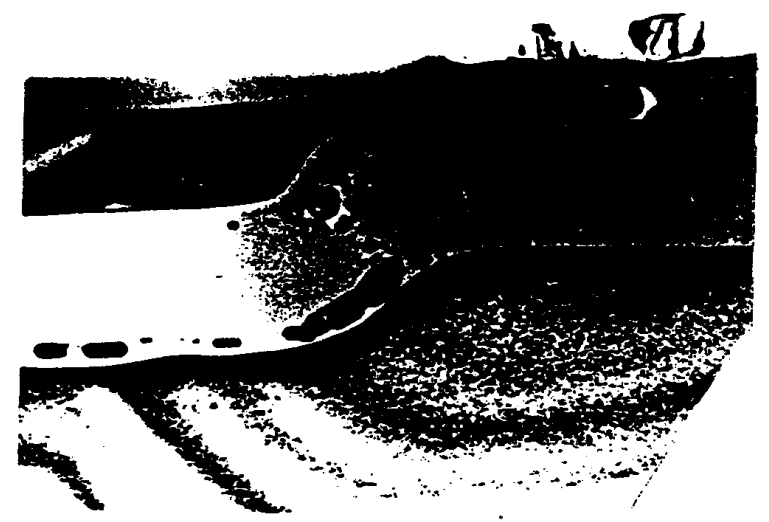

fig.1 Polycrystalline silicon mask: Periphery of window region (mask thickness $250 \mathrm{~nm}, 1.8 \times 10^{18} \mathrm{O}^{+} \mathrm{cm}^{-2}$ )

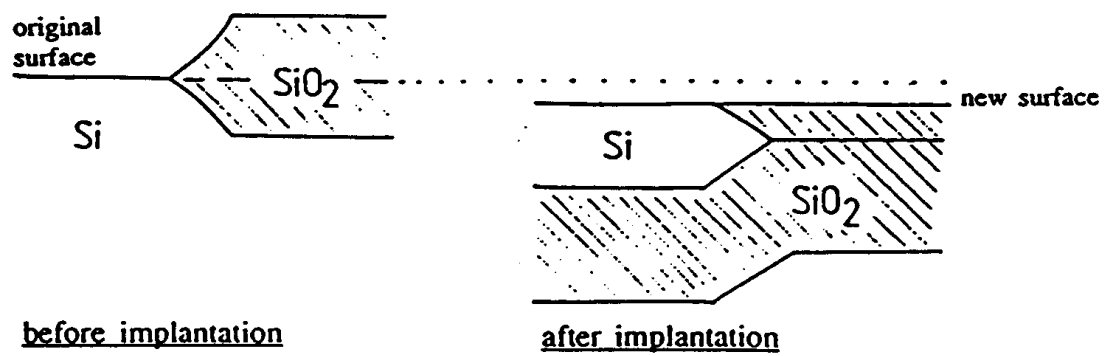

fig.2 LOCOS oxide mask (schematic): Expected structure of the periphery of the window region before and after implantation and annealing 